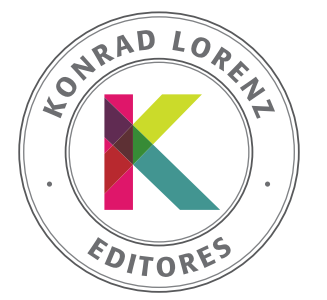

\title{
Interrogatorios y contrainterrogatorios en niños(as) testigos víctimas de delitos sexuales
}

\author{
Paola Rocío Bustos-Benítez y Olga Lucía Valencia Casallas*
}

Fundación Universitaria Konrad Lorenz, Bogotá, Colombia

Recibido el 4 de julio de 2018; aceptado el 29 de marzo de 2019

\section{PALABRAS CLAVE Testimonio infantil, interrogatorio, abuso sexual infantil, toma de decisiones judiciales}

\begin{abstract}
Resumen El testimonio infantil en delitos sexuales ha sido un tema investigado. Esta investigación tiene como objetivo, analizar las preguntas del interrogatorio y contrainterrogatorio, realizadas a niños(as) cuando rinden testimonio presumiéndose víctimas de delitos sexuales, bajo un estudio cualitativo con un diseño narrativo. Las unidades de análisis fueron las preguntas que hicieron la Fiscalía, Defensa, ministerio público y el juez en interrogatorio y contrainterrogatorio a niños(as) como víctimas en audiencias de juicio oral. Se contabilizaron 1126 preguntas, clasificadas en dos categorías orientadoras: tipos de preguntas y elementos de apoyo. Respecto a la primera, se generaron los siguientes códigos: preguntas frecuentes y necesarias, de hechos específicos, prohibidas e inadecuadas, aclaratorias del juez, preguntas de opinión del niño(a) y objetadas; la segunda categoría se codificó en los medios electrónicos, los juegos, dibujos anatómicos, láminas y videos. Los datos se analizaron con el software ATLAS.ti 7.5.4. La investigación demuestra que los actores judiciales deben considerar la forma en que hacen las preguntas para cumplir con los mínimos estándares legales, además de preservar el bienestar integral de los niños, niñas y adolescentes (NNA), por lo que se sugiere fomentar la formación en quienes interrogan.

(C) 2019 Fundación Universitaria Konrad Lorenz. Este es un artículo Open Access bajo la licencia CC BY-NC-ND (http://creativecommons.org/licenses/bync-nd/4.0/).
\end{abstract}

\section{Sexting among university students: Relation with age, sex and self-esteem}

Abstract The testimony of children in sexual crimes has been a subject investigated, but its study is not frequent when children are called as witnesses. The objective of this investigation was to analyze the interrogation and cross-examination questions made to children when they testify presuming victims of sexual crimes. It is a qualitative study, under a Narrative Design. The questions were obtained from eight videos of children giving testimony in oral hearing. The

\section{KEYWORDS}

Child testimony, interrogation, child sexual abuse, judicial decision making

\footnotetext{
* Autor para correspondencia.

Correo electrónico: olgaluciavalenciacasallas@gmail.com
} 
units of analysis were the questions asked by the Office of the Prosecutor, Defense, Public Prosecutor and the Judge in the practice of the cross-examination and cross-examination. There were 1126 questions, classified into two guiding categories: types of questions and support elements. The data was analyzed with Atlas Ti software. Regarding the types of questions, the following codes were generated: Frequent and necessary questions, questions of specific facts, forbidden and inadequate questions, clarifying questions of the Judge, questions of opinion of the child and questions objected. And regarding the support elements, electronic media, games, anatomical drawings, pictures and videos. The investigation shows that judicial actors must not only consider the way in which they ask questions, in order to comply with the minimum legal standards, but that the children be considered as a psychological subject, whose integral welfare should be preserved, so it is suggested to encourage training in those who question.

(c) 2019 Fundación Universitaria Konrad Lorenz. This is an open access article under the CC BYNC-ND license (http://creativecommons.org/licenses/bync-nd/4.0/).

La línea procesal penal en Colombia se operacionaliza bajo el Sistema Penal Oral Acusatorio (SPOA), el cual se norma en el Código de Procedimiento Penal (CPP), Ley 906 de 2004. En audiencia de juicio oral, para la toma del testimonio, en el artículo 391 se indica que los testigos serán interrogados por la parte que ofrece su testimonio como prueba (directo), luego la contraparte genera controversia (redirecto), bajo los hechos contestados previamente por estos. Las reglas para la realización del interrogatorio y del contrainterrogatorio, son determinadas en los artículos 392 y 393 del CPP.

Tabla 1 Reglas del interrogatorio y del contrainterrogatorio

\begin{tabular}{|c|c|}
\hline Reglas del interrogatorio & $\begin{array}{c}\text { Reglas del } \\
\text { contrainterrogatorio }\end{array}$ \\
\hline $\begin{array}{l}\text { Toda pregunta versará sobre } \\
\text { hechos específicos. }\end{array}$ & \multirow{5}{*}{$\begin{array}{l}\text { La finalidad es refutar, lo que } \\
\text { el testigo ha contestado. } \\
\text { Se puede utilizar cualquier } \\
\text { declaración que hubiese he- } \\
\text { cho el testigo sobre los hechos } \\
\text { en entrevista, en declaración } \\
\text { jurada durante la investiga- } \\
\text { ción o en la audiencia del jui- } \\
\text { cio oral. }\end{array}$} \\
\hline $\begin{array}{l}\text { El juez prohibirá preguntas su- } \\
\text { gestivas, capciosas o confusas. }\end{array}$ & \\
\hline $\begin{array}{l}\text { El juez prohibirá preguntas que } \\
\text { tiendan a ofender al testigo. }\end{array}$ & \\
\hline $\begin{array}{l}\text { El juez podrá autorizar al testi- } \\
\text { go para consultar documentos } \\
\text { necesarios que ayuden en su } \\
\text { memoria. }\end{array}$ & \\
\hline $\begin{array}{l}\text { El juez excluirá toda pregunta } \\
\text { que no sea pertinente. }\end{array}$ & \\
\hline
\end{tabular}

Teniendo en cuenta lo anterior y contextualizándolo en el escenario de interrogatorios en niños(as) en casos de delitos sexuales, Uribe (2011) refiere que es importante tomar el testimonio del niño(a) y cómo se vio afectado por este evento, ya que él es el principal afectado y por ello no debe ignorarse su testimonio. Tamara, Muñoz y Vergara (2012) refieren que el interrogatorio en estos casos, brinda información en el que se describen los hechos, por lo que su objetivo debe centrarse en obtener el testimonio de una manera pertinente, en donde se muestre la relación entre lo preguntado y la descripción de los hechos. Para ello, los actores procesales hacen preguntas al niño(a) por medio de un psicólogo, con el fin de ajustarlas con un lenguaje apropiado para no afectarlo, teniendo en cuenta la normatividad vigente (véase tabla 2).
Por tratarse de casos con menores de edad, además de hacer las preguntas que son sugeridas por la Fiscalía General de la Nación y por el CPP, se incluyen las de opinión del niño(a) según lo estipulado en el Código de Infancia y Adolescencia (Ley 1098 de 2006):

\section{Las preguntas de opinión del niño(a)}

La Ley 1098 de 2006, indica procedimientos especiales cuando los niños(as) son víctimas de delitos, y dispone especial atención para que en las diligencias en que intervengan se les tenga en cuenta su opinión, se les respete su dignidad, intimidad y demás derechos consagrados en esta ley. Igualmente, velará porque no se les generen nuevos daños con el desarrollo del proceso judicial. En los casos en que un niño(a) deba rendir testimonio, deberá estar acompañado de una autoridad especializada, de acuerdo con las exigencias contempladas en la ley.

\section{Las preguntas objetadas}

Las objeciones son el procedimiento utilizado para oponerse a la presentación de evidencia inadmisible, como también objetar una pregunta indebida durante el juicio. Se evidencia que las objeciones constituyen un mecanismo para establecer la contracción durante el juicio oral (Ortega, 2012), haciendo frente a aquellas preguntas que desvían la investigación hacia temas irrelevantes o inoportunos. De igual manera, las preguntas sugestivas o todas aquellas que puedan llegar a confundir al testigo pueden ser objetadas (Quiñones, 2003).

Por último, hay elementos de apoyo que se permiten para recabar el testimonio, como lo menciona el artículo 392-d del CPP: “El juez podrá autorizar al testigo para consultar documentos necesarios que ayuden a su memoria”. Entre ellos están (véase tabla 3).

En la práctica se observan errores en la realización de la entrevista que afectan el recuerdo, haciendo que la misma pierda su capacidad probatoria. Es importante hacer, a partir de un estudio sistemático, un análisis al tipo de preguntas y los elementos de apoyo en casos de denuncias de delitos sexuales, con el fin de aportar información científica para mejorar la forma en que se realizan preguntas en los contextos penales. 
Tabla 2 Tipos de preguntas realizadas

Preguntas frecuentes y necesarias

1. Preguntas de identificación y reconocimiento de la evidencia: dirigidas a que el testigo identifique y reconozca una evidencia que se pretende introducir con su testimonio (Fiscalía, 2009).

2. Preguntas de presentación del testigo: son las que ilustran al juez sobre la persona que va a deponer en la audiencia (Fiscalía, 2009).

3. Pregunta sobre hechos específicos: son las que ubican al testigo en las circunstancias de tiempo, modo y lugar, con respecto al caso (art. 392-a, CPP).

4. Preguntas de cierre: relacionadas con aquellos aspectos que tienen el propósito de vincular el testimonio con el acusado. Esto puede incluir un señalamiento de este en la sala de audiencias frente al juez, siempre que el testigo esté en capacidad de hacerlo (Fiscalía, 2009).

Preguntas prohibidas e inadecuadas (Código de Procedimiento Penal. Ley 906 de 2004)
1. Pregunta sugestiva: hace referencia a toda pregunta que por su forma y estructura sugiere al interrogado la respuesta; asimismo, en el interrogatorio no pueden realizarse preguntas que sugieran las respuestas, porque lo que se pretende es que el testigo declare abiertamente sobre los hechos. El juez prohibirá toda pregunta sugestiva, capciosa o confusa, con el fin de que el interrogatorio sea leal y que las respuestas sean claras y precisas (arts. 391 y 392-b, CPP).

2. Pregunta confusa: toda pregunta que no precisa el contenido de la información que se requiere, es incomprensible y distrae o confunde al testigo. Igualmente, el juez prohibirá este tipo de pregunta con el fin de que el interrogatorio sea leal y que las respuestas sean claras y precisas (art. 392-b, CPP).

3. Pregunta ofensiva: hace referencia a preguntas que tengan como finalidad generar malestar o predisposición con comentarios irrelevantes. El juez prohibirá toda pregunta que tienda a ofender al testigo (art. 392-c, CPP).

Prohibidas e inadecuadas / de oposición (Manual de Procedimientos de Fiscalía en el SPOA, 2009)
1. Pregunta impertinente o inconducente: es la que no tiene relación con los hechos debatidos en el juicio.

2. Pregunta conclusiva: pretende provocar en el testigo una respuesta que corrobore la conclusión que se formula con la pregunta sobre los hechos que se investigan.

3. Pregunta compuesta: abarca dos o más temas a la vez y crea confusión sobre cuál de los aspectos es el cuestionado.

4. Pregunta repetitiva: la que se hace al testigo de manera constante y en diferentes momentos del interrogatorio.

5. Pregunta especulativa: es aquella pregunta cuyo contenido no tiene soporte en los hechos que se investigan, ni en las pruebas producidas y conduce al testigo a que haga hipótesis o adivine, sin un soporte sobre lo que él percibió a través de sus sentidos.
Tienen la finalidad de aclarar alguna información que se expuso durante el interrogatorio o contrainterrogatorio por parte de la víctima. El artículo 397 del CPP señala: "Una vez finalizada los interrogatorios de las partes, el juez y el ministerio público podrán hacer preguntas complementarias para el cabal entendimiento del caso".

Tabla 3 Elementos de apoyo en la toma de declaración del niño(a)

\begin{tabular}{l|l}
\hline \multicolumn{1}{c|}{$\begin{array}{c}\text { Elementos } \\
\text { de apoyo }\end{array}$} & \multicolumn{1}{c}{ Definiciones } \\
\hline Láminas & Elementos impresos que pueden guiar al interrogado a dar respuestas más precisas. \\
\hline Videos & $\begin{array}{l}\text { Elemento audio-visual que contribuye al interrogado a evocar la información de manera más asertiva. Los } \\
\text { videos en asuntos periciales deben ser tenidos en cuenta en toda actividad relacionada con niños(as), ya que } \\
\text { se deteriora la validez de información si esta es sometida a excesivas pruebas y entrevistas, teniendo el fin } \\
\text { de minimizar el impacto de la actividad en los niños(as) y reconociendo la vulnerabilidad emocional a la que } \\
\text { es expuesto en cada una de ellas (Oficina de Defensoría de los Derechos de la Infancia, 2006). }\end{array}$ \\
\hline Juegos & $\begin{array}{l}\text { Actividades llevabas a cabo por una persona capacitada, cuyas finalidades están la de tener una mayor confianza } \\
\text { con el interrogado para que pueda evocar la información de manera adecuada (Tamara et al., 2012). }\end{array}$ \\
\hline Muñecos anatómicos & $\begin{array}{l}\text { Muñecos de tela suave con rasgos faciales, pelo y una representación identificable de los genitales externos } \\
\text { (Aldrídge, 1998). Pueden usarse para que el niño(a) identifique partes privadas del cuerpo o para saber si } \\
\text { sabe quién puede tocar los genitales (Cheung \& Boutté, 2010). }\end{array}$ \\
\hline Medios electrónicos & $\begin{array}{l}\text { Elementos impresos en los cuales se encuentren conversaciones realizadas mediante redes sociales que sean } \\
\text { pertinentes para ayudar a la memoria del interrogado (Odriozola \& Zunzunegui, 2008). }\end{array}$ \\
\hline
\end{tabular}




\section{Método}

\section{Unidades de análisis}

Para el estudio se tomaron la totalidad de las preguntas luego de transcribir ocho videos de niños(as) brindando testimonio en audiencia de juicio oral, cuyos criterios fueron los siguientes: el testigo tenía que ser solicitado por la Fiscalía, que durante el juicio se cumplieran las etapas de directo y redirecto y el caso debía haberse juzgado. Se contabilizaron 1.126 preguntas: 892 realizadas por la Fiscalía, 162 por la defensa, 44 por el ministerio público y 28 por el juez de conocimiento. Se clasificaron en dos categorías de estudio: tipos de preguntas y elementos de apoyo. Los datos se analizaron con el software ATLAS.ti 7.5.4. Para las trascripciones, se tomaron en cuenta las consideraciones éticas para conservar la confidencialidad de los niños(as) ${ }^{1}$.

\section{Diseño}

Se abordó desde un estudio cualitativo, bajo un diseño narrativo, en el cual "el investigador recolecta datos sobre experiencias de ciertas personas para describirlas y analizarlas. Se usa frecuentemente cuando el objetivo es evaluar una sucesión de acontecimientos" (Hernández, Fernández \& Baptista, 2014). Los autores señalan que los datos se obtienen de fuentes documentales, entrevistas y testimonios (que se encuentran en artículos en la prensa, grabaciones radiofónicas y televisivas, etc.).

\section{Procedimiento}

Inicialmente se hizo la transcripción de los ocho videos, posteriormente se dividieron las preguntas por los actores procesales para luego realizar la codificación de acuerdo con las dos categorías orientadoras: tipos de preguntas y elementos de apoyo. De la primera, se generaron los siguientes códigos: preguntas frecuentes y necesarias, preguntas de hechos específicos, preguntas prohibidas e inadecuadas, preguntas aclaratorias del juez, preguntas de opinión del niño y preguntas objetadas; de la segunda, los códigos fueron: medios electrónicos, los juegos, dibujos anatómicos, láminas y videos (véase tabla 4). Estos códigos se seleccionaron a partir de lo planteado en el Código de Procedimiento Penal, la Ley 1098 de 2006 y del Manual de Procedimientos de Fiscalía en el SPOA (2009).

\section{Resultados}

El análisis de los datos presenta que el $80 \%$ de las preguntas fueron realizadas por la Fiscalía, seguido de $15.1 \%$ de la defensa. A partir de las categorías orientadoras (tabla 4), se describen los hallazgos por cada una, incluyendo para cada código la fundamentación y la densidad; la primera es el número de apariciones en el discurso y la segunda, las veces que ese código se vincula con otros. Por ejemplo: preguntas de presentación del testigo (554-11), significa que se ha codificado 554 veces ese código y 11 hace referencia al número de códigos asociados.

Con relación a los códigos, la figura 1, preguntas frecuentes y necesarias (0-6), muestra que las categorías de mayor peso fueron: preguntas de presentación del testigo (554-11) y en segundo lugar, preguntas sobre hechos específicos (257-4).

De las preguntas de presentación del testigo (figura 2), se codificaron emergentes relacionadas con personas (23-1), lugares (9-1), rutinas (31-1), razones (49-1), gustos (5-1), el reconocimiento del concepto de verdad o mentira (13-1), de conocimiento sexual (11-2), discriminación mediante conceptos (12-3), conocimiento biológico (14-2) y conocimiento personal (239-7). Esta red semántica devela que estas preguntas pretenden "ilustrar al juez acerca de características relevantes de la persona que expondrá en audiencia", a este se le hacen más interpelaciones de las necesarias para ese fin. En la categoría emergente conocimiento personal (239-7) aparecen indagaciones que hacen que el sujeto dé información de datos personales (152-1), de tiempo y frecuencia de hechos (27-1), de aspectos del pasado (26-1), de detalles de cómo pasó el hecho (13-1) y de emociones (18-1) del niño(a). No obstante, aparecen preguntas que le exigen al niño(a) hacer distinciones entre actos, características de

Tabla 4 Categorías orientadoras

\begin{tabular}{|c|c|c|}
\hline Categorías orientadoras & Tipos & Fundamentación/Densidad \\
\hline Preguntas jurídicas & $\begin{array}{c}\text { Frecuentes y necesarias } \\
\text { Prohibidas e inadecuadas } \\
\text { Aclaratorias } \\
\text { De opinión del niño(a) } \\
\text { Objetadas }\end{array}$ & $\begin{array}{l}(0-6) \\
(0-11) \\
(0-3) \\
(0-4) \\
(0-10)\end{array}$ \\
\hline Elementos de apoyo & $\begin{array}{c}\text { Medios electrónicos } \\
\text { Juegos } \\
\text { Dibujos y muñecos anatómicos } \\
\text { Videos } \\
\text { Láminas }\end{array}$ & $\begin{array}{l}(18-1) \\
(0-1) \\
(4-1) \\
(0-1) \\
(0-1)\end{array}$ \\
\hline
\end{tabular}

1 En la Ley 1090 de 2006, tít. II, art. 2, num. 9, sobre la investigación con participantes humanos, se tuvo en cuenta el abordaje de la investigación respetando la dignidad y el bienestar de las personas que participan y con pleno conocimiento de las normas legales y de los estándares profesionales que regulan la conducta de la investigación con participantes humanos. En el tít. vil de la misma ley, art. 50, señala que los profesionales de la psicología al llevar a cabo investigaciones científicas, deberán basarse en principios éticos de respeto y dignidad, lo mismo que salvaguardar el bienestar y los derechos de los participantes, principio tenido en cuenta en esta investigación. La Ley 1098 de 2006, art. 33, señala: "Los niños(as) tienen derecho a la intimidad personal, mediante la protección contra toda injerencia arbitraria o ilegal en su vida privada, la de su familia, domicilio y correspondencia". 


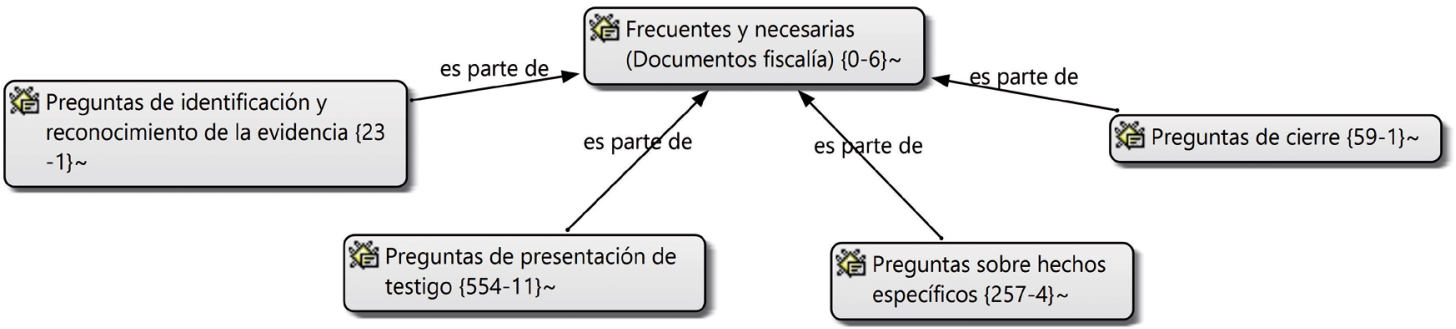

Figura 1 Preguntas frecuentes y necesarias

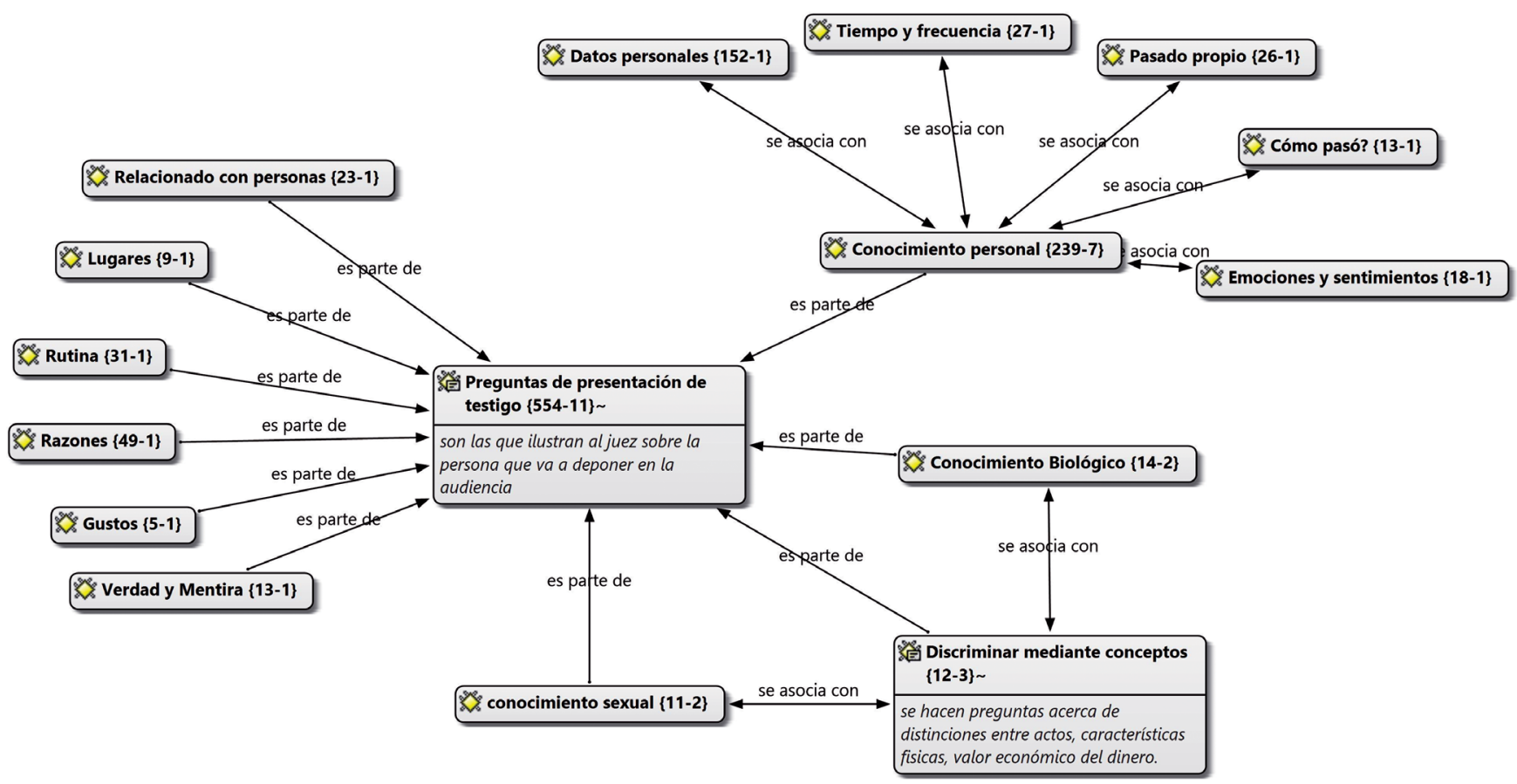

Figura 2 Preguntas de presentación de testigo

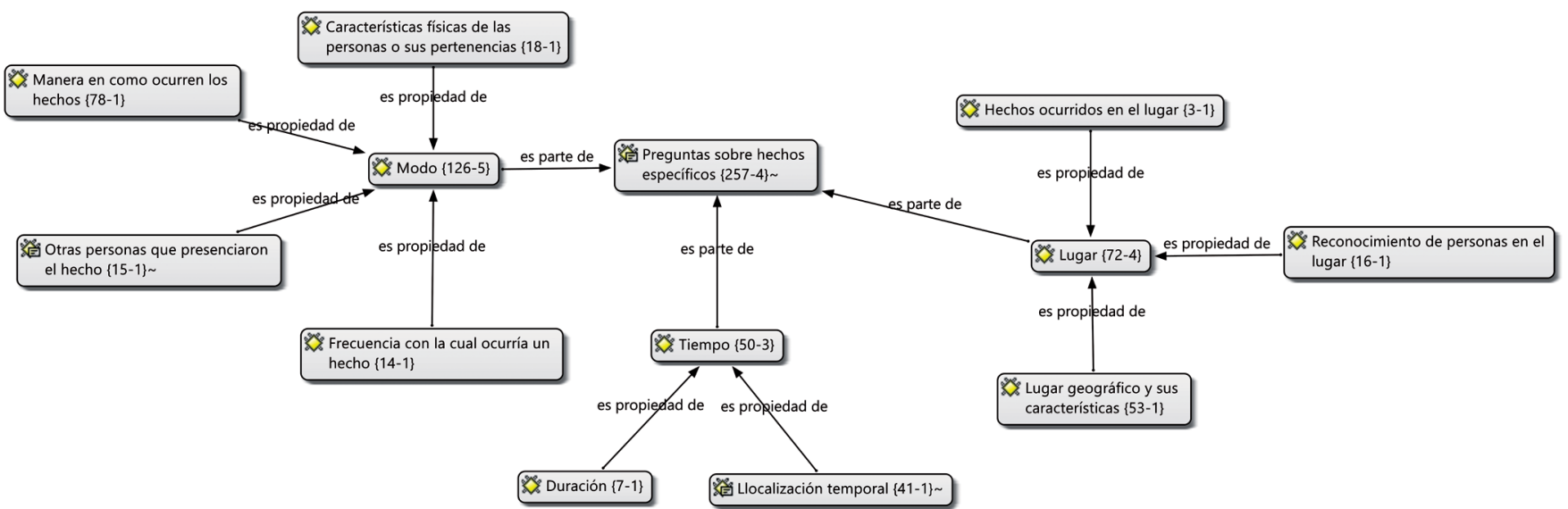

Figura 3 Preguntas sobre hechos específicos

objetos, lugares y personas, etc. Otras asociadas al conocimiento biológico y sexual, así como la constante solicitud de explicación de las razones (49-1) por las cuales suceden incidentes en la vida del niño(a) que, aunque pueden ser explicadas desde su perspectiva, están asociadas con acciones de terceras personas (23-1).
La figura 3 muestra las preguntas sobre hechos especificos (257-4), que apuntan a conocer las circunstancias de modo (126-5), tiempo (50-3) y lugar (72-4) de ocurrencia del supuesto abuso sexual. Respecto al modo, las interpelaciones que se hacen al testigo están dirigidas a que este responda por características propias o de otras personas, la 
representación detallada de los hechos y qué otras personas los presenciaron. En tiempo, se evidencia la duración de los hechos, descripción temporal (año, mes, día y hora). En cuanto a lugar, se hacen preguntas acerca de la ubicación geográfica y el reconocimiento de personas en el lugar. Respecto a las preguntas de cierre, es frecuente solicitar información que ya ha sido dada mediante otras preguntas, por ejemplo, se le indaga para que explique la forma en que se dan los actos inadecuados y quien los realiza.

En relación con las preguntas prohibidas $e$ inadecuadas (0-11) (figura 4), se resaltan las conclusivas (75-2). Hay una elevada frecuencia en que se pretende que el niño(a) dé respuestas que corroboren la conclusión formulada en la misma pregunta. La principal estrategia en estos casos es hacer preguntas dicotómicas (18-1), que ofrecen facilidad en las respuestas, porque el testigo solo tiene dos opciones de respuesta sin la necesidad de exponerse demasiado, por ejemplo: "Le puede preguntar, cuál fue el motivo, no había puerta en el baño o les tocaba bañarse sin cortina". Esto puede llegar a ser muy atractivo para él, y conducir a que sea el tipo de preguntas que prefiera responder. Por otra parte, los resultados en las preguntas especulativas (30-1) complementan la anterior afirmación, dado que no se argumentan ni en las pruebas, ni en los hechos investigados. En la tabla 5 se presentan otros tipos de preguntas de este código.

Entre las preguntas aclaratorias del juez (figura 5), aparecen las relacionadas con derechos en la declaración (2-1), mediante las cuales se explican implicaciones legales que puedan contextualizar al niño(a) de límites y posibilidades que tiene al momento de declarar.

Se realizan nuevamente preguntas de identificación (142 ), tendientes a conocer información básica del niño(a), centrándose esta vez en obtener datos de las dimensiones familiar y educativa. Se indaga por la percepción que el niño(a) tiene de la forma en que le manifiestan afecto sus



Figura 4 Preguntas prohibidas e inadecuadas

Tabla 5 Tipos de preguntas

\begin{tabular}{|c|c|c|}
\hline Preguntas prohibidas & Densidad & Cita \\
\hline Pregunta conclusiva & $75-2$ & "Y aún así no les ha vuelto a hablar". \\
\hline Pregunta especulativa & $30-1$ & $\begin{array}{l}\text { "Por qué decir que X la había violado y no otra persona por qué le surgió mencionar } \\
\text { a X en ese momento". }\end{array}$ \\
\hline Pregunta compuesta & $19-1$ & “Has hablado con tu tío en estos días, últimamente. Qué te ha dicho". \\
\hline Pregunta repetitiva & $18-1$ & $\begin{array}{l}\text { “¿Le preguntamos a la menor si ella recuerda bien?, vamos a tratar de establecer } \\
\text { el proceso de rememoración de la niña, ¿sí recuerda cosas que han sucedido el año } \\
\text { pasado? ¿Cómo está ubicada la menor en el tiempo? ¿La menor podrá recordar si el } \\
\text { año pasado alguien le tocó su vagina?”. }\end{array}$ \\
\hline $\begin{array}{l}\text { Pregunta confusa, ambigua, } \\
\text { vaga, inteligible }\end{array}$ & $16-1$ & $\begin{array}{l}\text { "Pregúntele si se acuerda cómo era la profesora, que nos trate de decir su estado, si } \\
\text { estaba bien o no estaba bien". }\end{array}$ \\
\hline $\begin{array}{l}\text { Pregunta impertinente o } \\
\text { inconducente }\end{array}$ & $11-1$ & “El día que ocurrió eso, qué había comido, si había desayunado y almorzado". \\
\hline Pregunta sugestiva & $3-1$ & “¿X le dijo algo antes de tirarla a la colchoneta?”. \\
\hline Pregunta ofensiva & $2-1$ & $\begin{array}{l}\text { “Cuando el señor } X \text { le solicitaba o le pedía a usted que le chupara el pene, ¿cuál era } \\
\text { su reacción?... ¿Entonces lo hacía o no lo hacía?... ¿En qué posición estaba usted?”. }\end{array}$ \\
\hline
\end{tabular}


padres, qué tanto se involucraban en el aseo personal, lo que se denomina en la figura conducta de los padres (3-3). Finalmente, emerge el código detalles de los hechos que indaga acerca de la manera en que el niño(a) se refiere al acusado, qué tanto lo conoce y detalles específicos de los hechos (7-2) antes, durante y después.

En las preguntas de opinión del niño(a), emergen códigos relacionados con los hechos (25-1) y con lo que sucede con el niño(a) en la audiencia (6-1). Estos resultados se analizan teniendo como premisa el cuidado que se ha de tener para prevenir que el proceso jurídico se convierta en una situación aún más aversiva, que el mismo hecho. En este marco se compararon las densidades de las preguntas inadecuadas (0-11), con las relacionadas con los hechos (25-1) y las que indagan acerca de lo que está sucediendo en el interrogado en la audiencia (6-1), pudiendo afirmarse que no se tiene como prioridad el cuidado de la experiencia psicológica del niño(a) en esta.

En la figura 6 están las preguntas objetadas (0-10). Se encontró que las conclusivas (24-1), las impertinentes $o$ inconducentes (15-1) y las especulativas (13-1), son las más utilizadas.
Aunque se consideró la posibilidad que en el juicio se indagara por dibujos anatómicos (4-1), juegos (0-1), láminas (0-1), medios electrónicos (18-1) y videos (0-1), solo se preguntó por medios electrónicos y dibujos anatómicos (véase figura 7).

\section{Discusión}

Los resultados permitieron evidenciar la dinámica de los actores procesales en los interrogatorios y los contrainterrogatorios cuando tienen como testigos a niños, niñas y adolescentes (NNA) en casos de presuntos delitos sexuales. Es notorio el afán porque los NNA respondan de manera lógica y sistemática, el porqué de una serie de sucesos relacionados con el presunto abuso o sus consecuencias en el ámbito familiar, personal o social. Hay evidencia sobre la relación entre el proceso judicial y la situación de abuso que sufre la víctima, presentándose consecuencias en la regulación de sus emociones, el apego, el funcionamiento cognitivo y conductual (Judicial Council of California, 2014), y más adelante, conductas de riesgo sexual mediadas por el uso de

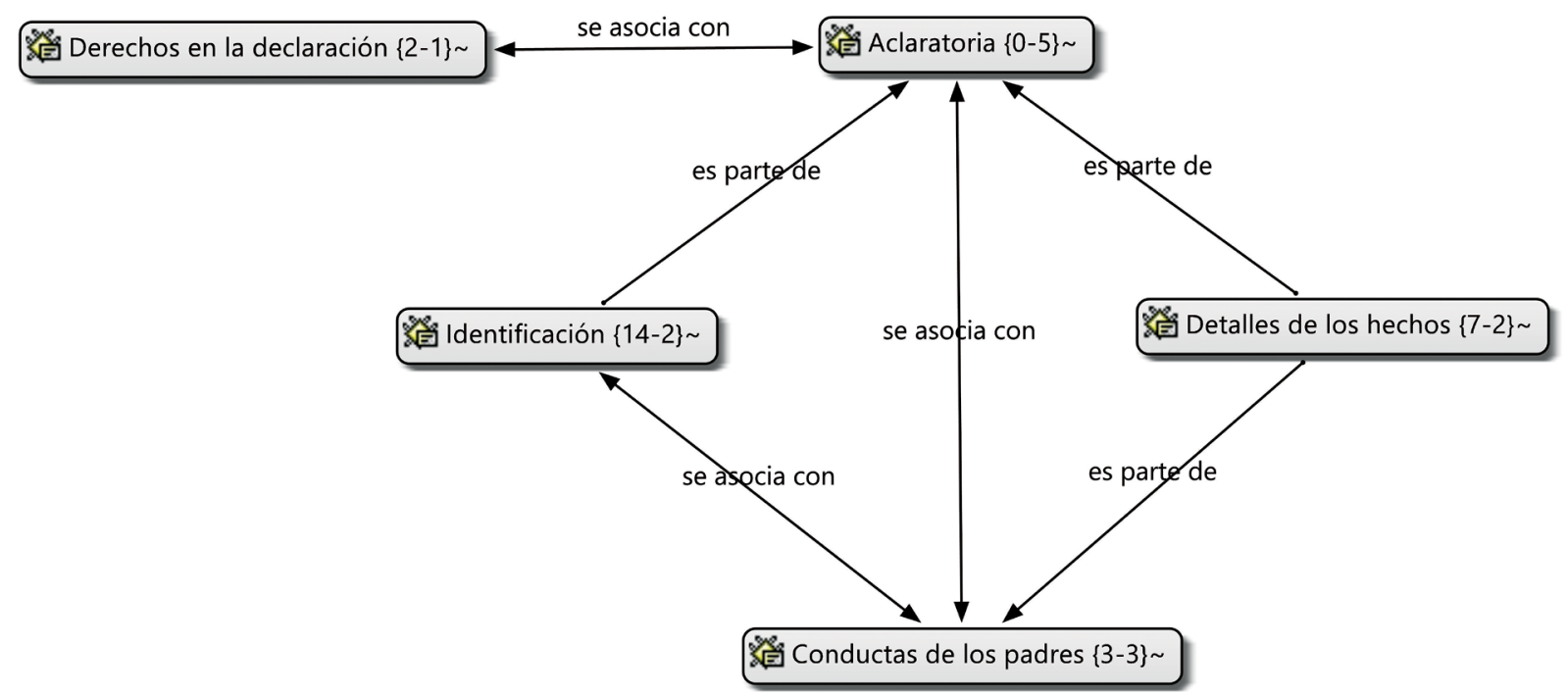

Figura 5 Preguntas aclaratorias del juez

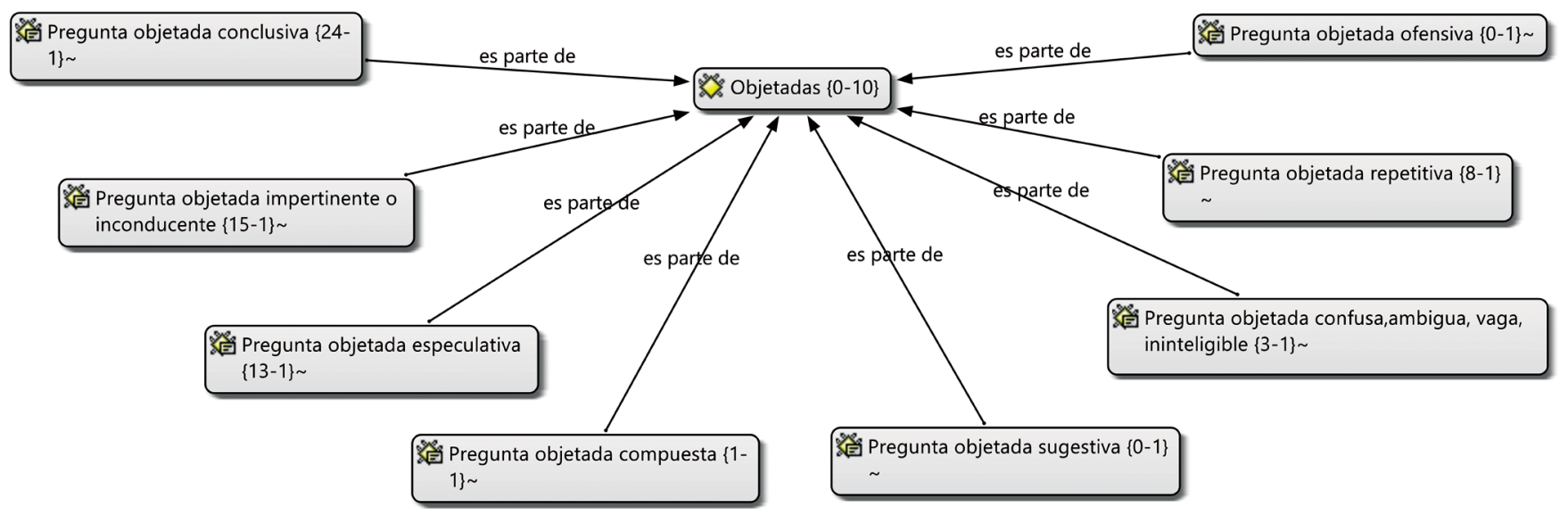

Figura 6 Preguntas objetadas 


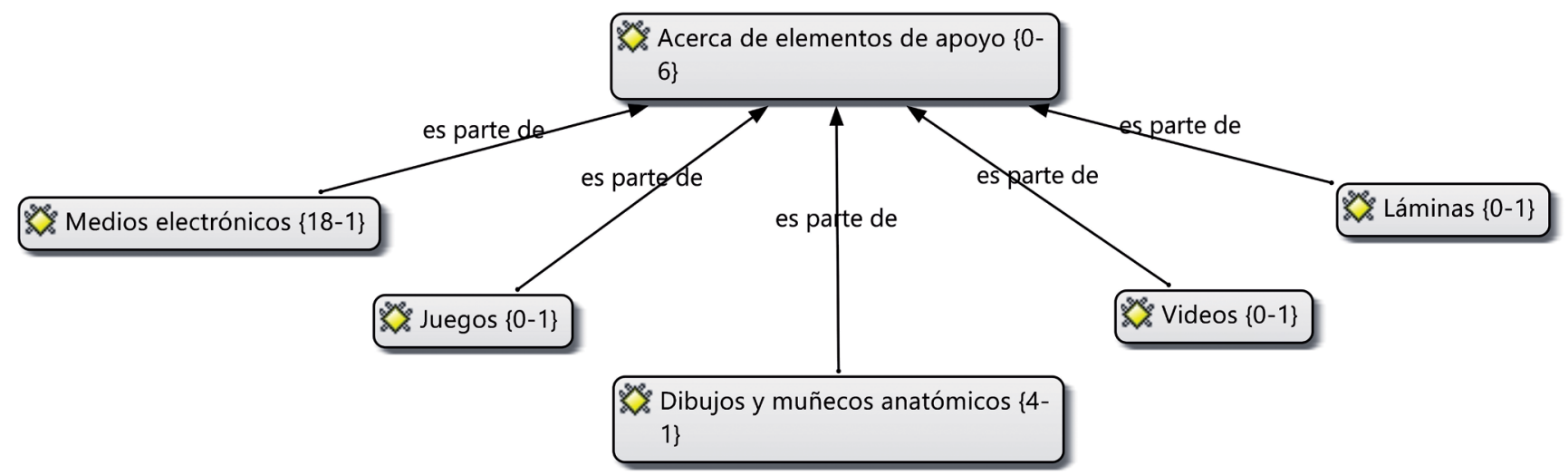

Figura 7 Preguntas acerca de elementos de apoyo

alcohol y cigarrillo (Yahaya, Ponce, Uthman, Soares y Macassa, 2015). Se puede afirmar que toda la actividad de evocación y afrontamiento de situaciones, eventos, personas o recuerdos relacionados con el abuso, están determinadas o se ven alteradas no solo por la etapa del desarrollo en la que se encuentra el niño(a), sino también por el hecho de que la situación de abuso, así como sus consecuencias escaparon de su control. No obstante, existe consenso en la literatura en que la proporción de falsos relatos de abuso sexual efectuados por menores es pequeña (Dos Santos \& De Oliveira, 2016), lo cual confirma que los niños(as) tienden a relatar hechos reales aun en medio de las adversidades expuestas hasta el momento y debe ser compromiso de todos los actores del proceso judicial, aliviar esa carga, para que se consiga el mismo resultado con menor nivel de riesgo, evitando la victimización secundaria (Townsend, Waterhouse \& Nomdo, 2014), la cual sucede no como resultado directo de la acción delictiva, sino por la respuesta de las instituciones e individuos específicos hacia la víctima (Oficina de las Naciones Unidas contra la Droga y el Delito, 2009).

Según Subijana y Echeburúa (2018), la forma como se juzga exige una justicia específica para los menores cuando son víctimas, y es necesario que se tenga en cuenta el derecho del menor a que su interés superior sea valorado y considerado como primordial en todas las actuaciones judiciales, y la necesidad de que en las audiencias se tenga en cuenta su desarrollo evolutivo. Esto se logra con la asistencia de profesionales cualificados y expertos que preserven su intimidad y utilicen un lenguaje que le sea comprensible.

Igualmente, para garantizar el bienestar del testigo de los NNA, es a este al que debería ayudársele a resolver las dudas que tenga y no transmitirle directa o indirectamente la responsabilidad de analizarlas o comprenderlas; según Murray, Nguyen y Cohen (2014), el abuso sexual infantil no suele ser un problema de corto plazo, por tanto es necesario que se apoye al niño(a) en distintos momentos con el fin de prevenir daños mayores. Los hallazgos indican que las personas expuestas a este tipo de abusos, durante la adolescencia pueden ser particularmente vulnerables a trayectorias de revictimización crónica, caracterizadas por múltiples índices de riesgo (Papalia et al., 2017).

Otros aspectos que se exigen en las diligencias judiciales son: se debe realizar una sola entrevista con profesionales especializados, de modo que el niño(a) no tenga que reiterar su declaración y recordar todo el trauma padecido; se deben formular preguntas claras, directas y de estructura sencilla, relacionadas con la materia investigada (Ulfe, 2015); el interrogatorio debe adaptarse al léxico del niño(a), integrado por preguntas abiertas que permitan un relato fluido, sin que sean admisibles preguntas directivas, sugestivas o inductivas, aspecto que no se evidencia en los resultados de esta investigación. No cabe formular juicios, ni críticas, para no influir en la calidad del testimonio, afirmaciones o actitudes del entrevistador (Echeburúa \& Subijana, 2008).

Cuando las preguntas se centran en los hechos específicos, el nivel de detalle que tiene que dar el niño(a) lo conduce nuevamente a enfrentarse al evento, evocando situaciones concretas, lo que traerá reacciones psicológicas desagradables. Para esto, es necesario el apoyo social, que según Moraru (2012), citando a Mardare (2010), facilita la integración del individuo en la sociedad. De allí, la necesidad de pensar en la formación de actores legales para garantizar características personales, que faciliten la percepción de apoyo en la relación con el testigo, más si se tiene en cuenta que el apoyo social representa un factor protector contra numerosas formas de abuso.

Ahora bien, los niveles de detalle exigidos en la evocación de tiempo, modo y lugar del hecho pueden llegar a ser intrusivos para el niño(a), pero también existe la posibilidad que producto de la exposición constante, el relato de la situación de abuso sexual infantil se normalice, llegando a hablar de esto en contextos de poco control, como el salón de clases, aumentando el riesgo de recibir recriminaciones por su experiencia. Como afirma Hlavka (2008), la interpretación y la divulgación del abuso sexual están estructuradas por las prácticas y grupos sociales en las que los NNA participan y por eso es necesario tener en cuenta que factores como la estigmatización, culpa o temor a las consecuencias, se convierten en limitantes para la interacción. Cabe también la posibilidad de que la experiencia en el juicio sea tan aversiva, que el testigo recurra a suprimir, modificar o desistir de hablar de la situación.

De las preguntas prohibidas e inadecuadas se resaltan las conclusivas. Puede verse la elevada frecuencia con que se pretende que el niño(a) dé respuestas que corroboren la conclusión formulada en la misma pregunta. La principal estrategia en estos casos es hacer preguntas dicotómicas que ofrecen facilidad en la respuesta por parte del testigo. Los resultados observados en las preguntas especulativas, permiten concluir que se dirige la atención del testigo a la construcción de hipótesis y no a la percepción inmediata de 
los hechos, sin procesos cognitivos adicionales. En relación con esto, Gagnon y Cyr (2017) sugieren que el uso de preguntas abiertas ayuda a proporcionar un relato detallado del supuesto abuso sexual. Finalmente, hay una baja recurrencia a las preguntas ofensivas y sugestivas debido a que son fácilmente detectables y objetables.

Según Fontes \& Tishelman (2016), las entrevistas forenses con niños(a) por supuesto abuso sexual requieren ubicar a los niños(as) “donde están” en términos de su nivel de desarrollo, disposición a revelar, su cultura y lenguaje. Por otro lado, en el escenario donde el juez requiere ampliar la información, la mayor cantidad de información recabada por este, está relacionada con los hechos; pero un análisis más profundo de la relación entre las categorías emergentes, permite observar la tendencia a indagar sobre si estos se trataron de hechos aislados o si fueron producto de una acción planeada, utilizando espacios que serían de exclusividad del sistema parento-filial como son las rutinas de cuidado y autocuidado en el aseo personal. No es frecuente que se le consulte por las emociones o pensamientos de lo que le está ocurriendo en el momento de la audiencia, sino que la tendencia es a extraer información de manera inadecuada.

En el marco de las preguntas objetadas, es importante observar la funcionalidad que tienen estas para la contraparte, de modo que durante la audiencia sirva para que el testigo se confunda, distorsione o imagine nuevos datos, por cuanto se le formulan conclusiones dentro de la misma pregunta, para que las corrobore. También puede llevar a que el niño(a) adivine o realice hipótesis, pero no a describir lo que realmente percibió.

En cuanto a los elementos de apoyo, en la investigación se encontró que solo se les preguntó por medios electrónicos, dibujos y muñecos anatómicos. Pero no es la falta de preguntas por estos elementos lo que se destaca, sino que, en tiempos de desarrollo tecnológico y académico, en estos procesos no se cuenta con gran variedad de recursos didácticos que permitan recolectar la información de manera acorde con la edad del testigo.

Finalmente, podría concluirse que en este tipo de prácticas en escenarios adversariales con los NNA, existen un sujeto jurídico y uno psicológico que convergen en el testigo, los dos tratados de manera diferente en el marco del cumplimiento de la normatividad. El primero goza de garantías procesales, en la que los mecanismos de protección se activan como instrumentos objetivos que se materializan en la presencia de personas que representan estamentos, que nunca tendrán contacto directo con el testigo. Esta serie de elementos, que se ofrecen como garantías del respeto por los derechos superiores del niño(a), solo median para entrar en contacto con el sujeto psicológico. El segundo sujeto referido a la experiencia psicológica del niño(a), se activa en el marco de una relación legal que, si bien pretende establecer la verdad, justicia y reparación, no se compara a una relación formal de ayuda de la cual este sea consciente.

Este artículo buscar señalar la importancia de un equilibrio cuando se planeen las preguntas conforme a la ley, de modo que se cumpla el doble objetivo: la obtención de la información, contemplando a la vez el bienestar del niño(a). Los anteriores hallazgos demuestran una clara necesidad para generar acciones de capacitación a los actores judiciales, de modo que se planeen de manera anticipada las preguntas que se realizan, siguiendo las reglas jurídicas, pero a la vez siguiendo los lineamientos que se plantean desde el Código de Infancia y Adolescencia, donde se promueve la protección integral de los derechos de los NNA.

Por último, las preguntas en este estudio se analizaron desde lo jurídico, por lo que es relevante explicar el contenido de los interrogatorios, analizando las variables psicológicas que puedan estar involucradas (la cognición, la memoria, los estados afectivos, entre otros), las cuales afectan de igual manera las respuestas dadas en audiencia. Como lo señala la Agencia de los Derechos Fundamentales de la Unión Europea (2015) si los sistemas judiciales estuvieran mejor adaptados a la infancia, los niños(as) estarían más protegidos, podrían participar de manera más efectiva y se mejoraría, a la vez, el funcionamiento de la justicia.

\section{Referencias}

Agencia de los Derechos Fundamentales de la Unión Europea (FRA) (2015). Informe sobre la Justicia adaptada a la infancia: perspectivas y experiencias de los profesionales. Recuperado de https://fra.europa.eu/sites/default/files/fra-2015-child-friendly-justice-professionals-summary_es.pdf

Aldrídge, N. (1998). Strengths and limitations of forensic child sexual abuse interviews with anatomical dolls: An empirical review. Journal of Psychopathology and Behavioral Assessment, 1-41.

Barreto, I., Velandia-Morales, A., \& Rincón-Vásquez, J. (2011). Estrategias metodológicas para el análisis de datos textuales: aplicaciones en psicología del consumidor. Suma Psicológica, 18(2), 7-15.

Cheung, M., \& Boutté, Q. N. (2010). Assessing the relative importance of the child sexual abuse interview protocol items to assist child victims in abuse disclosure. Journal of Family Violence, 11-22.

Congreso de la República. (2004). Ley 906 de 2004. Código de Procedimiento Penal. Recuperado de http://www.alcaldiabogota. gov.co/sisjur/normas/Norma1.jsp?i=14787.

Congreso de la República. (2006). Ley 1098 de 2006. Código de la Infancia y la Adolescencia. Recuperado de http://www.alcaldiabogota.gov.co/sisjur/normas/Norma1.jsp?i=22106.

Dos Santos, S. W., \& De Oliveira, B. J. (2016). Characteristics of children under 12 years subjected to forensic examination on suspicion of child sexual abuse in Salvador Brazil. Spanish Journal of Legal Medicine, 55-61. http://dx.doi.org/10.1016/j. remle.2015.06.003

Echeburúa, E., \& Subijana, I. J. (2008). Guía de buena práctica psicológica en el tratamiento judicial de los niños abusados sexualmente. International Journal of Clinical and Health Psychology, 8(3), 733-749.

Fiscalía General de la Nación. (2009). Manual de procedimientos de la Fiscalía en el Sistema Penal Acusatorio. Bogotá.

Fontes, L., \& Tishelman, A. (2016). Language competence in forensic interviews for suspected child sexual abuse. Child Abuse \& Neglect, 51-62. http://dx.doi.org/10.1016/j.chiabu.2016.06.014

Gagnon, K., \& Cyr, M. (2017). Sexual abuse and preschoolers: Forensic details in regard of question types. Child Abuse \& Neglect, 109-118. http://dx.doi.org/10.1016/j.chiabu.2017.02.022

Hernández, R., Fernández, C. y Baptista, P. (2014). Metodología de la investigación. Editorial Mc Graw Hill. México.

Hlavka, H. (2008). The trouble with telling: Children's constructions of sexual abuse (tesis doctoral de Filosofía). Universidad de Minnesota, Minnesota. Recuperado de https://search-proquestcom.ezproxyucdc.ucatolica.edu.co/criminaljusticeperiodicals/ docview/304533169/D1D30A5AA77A4B62PQ/1?accountid=45660.

Judicial Council of California. (junio de 2014). California Courts. Obtenido de California Courts website: http://www.courts.ca. gov/documents/effects-complex-trauma-on-youth-briefing.pdf. 
Moraru, E. (2012). Childhood abuse experience: The effects on a child's personality and the role of social support. Revista de Asistenta Sociala; Bucharest, 35-49.

Murray, L., Nguyen, A., \& Cohen, J. (2014). Child sexual abuse. Child and Adolescent Psychiatric Clinics of North America, 321337. http://dx.doi.org/10.1016/j.chc.2014.01.003

Odriozola, E., \& Zunzunegui, I. (2008). Guía de buena práctica psicológica en tratamiento judicial de los niños abusados sexualmente. International Journal of Clinical and Health Psychology, 8(3), 733-749.

Oficina de Defensoría de los Derechos de la Infancia. (2006). Acciones para evitar la revictimización del niño víctima del delito: manual para acompañar a niños a través de un proceso judicial. Recuperado de http://www.inm.gob.mx/static/Autorizacion_ Protocolos/SSP/Tomo\%20_IV_Acciones_para_evitar_la_revictimizacion_del_niNo.pdf.

Oficina de las Naciones Unidas contra la Droga y el Delito. (2009). La justicia en asuntos concernientes a menores víctimas y testigos de delitos. Ley modelo y comentarios. V.09-85206, Viena (Austria). Recuperado de https://www.unodc.org/documents/ justice-and-prison-reform/Justice_in_matters_ES.pdf

Ortega, W. (2012). Litigación oral en el proceso penal. Santiago de Chile: Ril.

Papalia, N., Luebbers, S., Ogloff, J., Cutajar, M., Mullen, P., \& Mullen, P. (2017). Further victimization of child sexual abuse victims: A latent class typology of re-victimization trajectories. Child Abuse \& Neglect. http://dx.doi.org/10.1016/j.chiabu.2017.02.040

Pelaprat, M. (2009). Complex trauma among court-involved youth (tesis doctoral en Psicología). Massachusetts School of Professional Psychology \& University of California, Massachusetts.

Quiñones, H. (2003). Las técnicas de litigación legal en el proceso penal salvadoreño. San Salvador.

Strauss, A., \& Corbin, J. (2002). Bases de la investigación cualitativa. Técnicas y procedimientos para desarrollar la teoría fundamentada. Medellín, Antioquia, Colombia: Universidad de Antioquia.
Subijana, I. J., \& Echeburúa, E. (2018). Los menores víctimas de abuso sexual en el proceso judicial: el control de la victimización secundaria y las garantías jurídicas de los acusados. Anuario de Psicología Jurídica, 28(1), 22-27. http://dx.doi.org/10.5093/ apj2018a1

Tamara, M., Muñoz, P., \& Vergara, V. (2012). Diseño de un protocolo dirigido a la toma del interrogatorio y contrainterrogatorio de niños y niñas con edades comprendidas entre los 3 y los 7 años, víctimas del delito de acto sexual con menor de 14 años. Revista Vanguardia Psicológica Clínica Teórica y Práctica, 2(2), 216-231.

Townsend, L., Waterhouse, S., \& Nomdo, C. (2014). Court support workers speak out: Upholding children's rights in Upholding children's rights in. SA Crime Quarterly, (48), 74-88. http://dx.doi. org/10.4314/sacq.v48i1.7

Ulfe, E. (2015). Tecnología que evita la revictimización en niños, niñas y adolescentes: cámara gesell (Technology that prevents re-victimization in children and adolescents: camera gesell). $H A$ MUT'AY, 2(2), 58-66. http://dx.doi.org/10.21503/hamu.v2i2.916

Ulrich, H. (2007). Examining the variability in the long term adjustment of child sexual abuse victims (tesis doctoral en Psicología Clínica). Universidad de Montana. Missoula, Montana.

Uribe, N. (2011). Abuso sexual infantil y administración de justicia en Colombia. Reflexiones desde la psicología forense. Pensamiento Psicológico, 9(16), 183-202.

Yahaya, I., Ponce, D. L., Uthman, O., Soares, J., \& Macassa, G. (2015). Childhood sexual abuse among girls and determinants of sexual risk behaviours in adult life in sub-Saharan Africa. Journal of Aggression, Conflict and Peace Research, 67-75. http:// dx.doi.org/10.1108/JACPR-04-2014-0121 